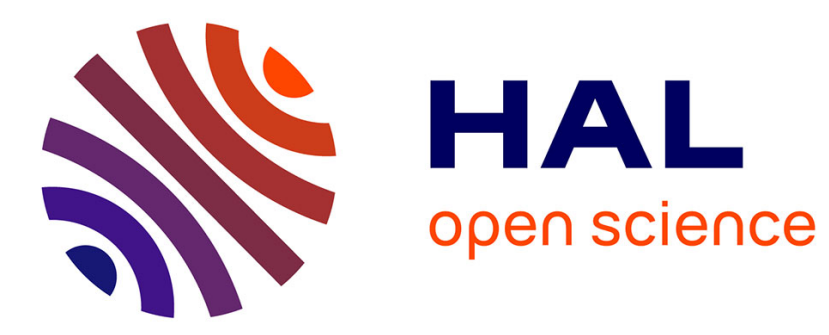

\title{
Finite-Time Ruin Probabilities for Discrete, Possibly Dependent, Claim Severities
}

\author{
Stéphane Loisel, Claude Lefèvre
}

\section{To cite this version:}

Stéphane Loisel, Claude Lefèvre. Finite-Time Ruin Probabilities for Discrete, Possibly Dependent, Claim Severities. Methodology and Computing in Applied Probability, 2009, 11 (3), pp.425-441. 10.1007/s11009-009-9123-9 . hal-00201377

\section{HAL Id: hal-00201377 https://hal.science/hal-00201377}

Submitted on 28 Dec 2007

HAL is a multi-disciplinary open access archive for the deposit and dissemination of scientific research documents, whether they are published or not. The documents may come from teaching and research institutions in France or abroad, or from public or private research centers.
L'archive ouverte pluridisciplinaire HAL, est destinée au dépôt et à la diffusion de documents scientifiques de niveau recherche, publiés ou non, émanant des établissements d'enseignement et de recherche français ou étrangers, des laboratoires publics ou privés. 


\title{
Institut de Science Financière et d'Assurances
}

\section{Les Cahiers de Recherche de I'ISFA}

\author{
FINITE-TIME HORIZON RUIN PROBABILITIES \\ FOR INDEPENDENT OR DEPENDENT CLAIM AMOUNTS
}

Claude Lefèvre

Stéphane Loisel

Cahier de Recherche WP 2045 (2007)

Université Claude Bernard Lyon 1

Retrouvez la liste complète des Cahiers de recherche de l'ISFA à l'adresse :

http://isfaserveur.univ-lyon1.fr/cahiers-recherche-isfa/ 


\title{
Finite Horizon Ruin Probabilities for Independent or Dependent Claim Amounts
}

\author{
CLAUDE LEFÈVRE \\ clefevre@ulb.ac.be \\ Département de Mathématique, Université Libre de Bruxelles, Campus de la Plaine C.P. \\ 210, B-1050 Bruxelles, Belgium \\ STÉPHANE LOISEL \\ stephane.loisel@univ-lyon1.fr \\ Université de Lyon, Université Claude Bernard Lyon 1, Institut de Science Financière et \\ d'Assurances, 50 Avenue Tony Garnier, F-69007 Lyon, France
}

\begin{abstract}
This paper is concerned with the compound Poisson risk model and two generalized models with still Poisson claim arrivals. One extension incorporates inhomogeneity in the premium input and in the claim arrival process, while the other takes into account possible dependence between the successive claim amounts. The problem under study for these risk models is the evaluation of the probabilities of (non-)ruin over any horizon of finite length. The main recent methods, exact or approximate, used to compute the ruin probabilities are reviewed and discussed in a unified way. Special attention is then paid to an analysis of the qualitative impact of dependence between claim amounts.
\end{abstract}

Keywords: compound Poisson model, ruin probability, finite-time horizon, recursive methods, (generalized) Appell polynomials, non-constant premium, non-stationary claim arrivals, interdependent claim amounts, impact of dependence, comonotonic risks, heavytailed distributions

AMS 2000 Subject Classification: 62P05, 60G40, 12E05

\section{Introduction}

A central model in insurance risk theory is the compound Poisson model. Since the pioneering works of Lundberg and Cramér, it has been the object of a number of theoretical studies and practical applications. Much on these questions can be found in the comprehensive books by Asmussen [2], Dickson [11], Gerber [14], Grandell [15], Kaas et al. [19], Panjer and Willmot [25] and Rolski et al. [29].

In its classical version, the compound Poisson model is concerned with a large portfolio of insurance policies, all independent and each with a small constant probability of undergoing a claim. This situation is quite realistic for many situations and it explains (in part) the great success of the model. In certain other cases, however, such assumptions may be found too restrictive. Nevertheless, an advantage of the model is that additional 
economic factors can be incorporated while preserving the relative simplicity of its analysis. Our purpose in the present paper is to point out this merit of the model within the framework of the evaluation of the (non-)ruin probabilities over any finite-time horizon.

Starting with the classical model, we present in Section 2 a review of the different methods that are proposed in the literature to calculate or approximate the finite-horizon ruin probabilities. Most of these methods are rather well-established; see, e.g., the books referred to above and the papers by De Vylder and Goovaerts [8], Dickson and Waters [12], Picard and Lefèvre [27], De Vylder [7], Rullière and Loisel [30] and Lefèvre and Loisel [21]. Section 3 discusses a first extended model that takes into account non-constancy in the premium input and non-stationarity in the claim arrival process. The results given here are probably less standard and come mainly from recent works by Picard and Lefèvre [27], Ignatov and Kaishev [16], Ignatov et al. [18], Lefèvre and Picard [22] and Lefèvre [20]. Section 4 is concerned with a second generalization in which the successive claim amounts are now allowed to be interdependent. This part is directly inspired by the works of Ignatov and Kaishev [16], [17] and Ignatov et al. [18]. We will follow, however, a slightly different and simpler approach that will enable us to derive an alternative expression, with an underlying polynomial structure, for the non-ruin probabilities. In Section 5, the qualitative impact of dependence is investigated to some extent. In particular, it is shown that for certain heavy-tailed claim amounts, positive dependence can either increase or decrease ruin probabilities when the initial surplus is large. Such a result is loosely related to those obtained, e.g., by Wüthrich [34] and Albrecher et al. [1].

To close, let us mention that the analysis made here can be adapted to the compound binomial risk model, a discrete-time analogue of the compound Poisson model.

\section{Classical compound Poisson model}

An insurance company has an initial surplus $u \geq 0$ and receives premiums continuously at a constant rate $c>0$. Claims arise according to a homogeneous Poisson process $\{N(t)\}$ with mean $\lambda$ per unit time, and, independently of this process, the successive claim amounts $\left\{X_{i}\right\}$ are non-negative independent and equidistributed random variables, with common distribution function $F(x)$. So, the aggregate claims constitute a compound Poisson process $\{S(t)\}$ where $S(t)=\sum_{i=1}^{N(t)} X_{i}$. The surplus at time $t$ is then given by

$$
U(t)=u+c t-S(t)
$$

and ruin occurs as soon as the surplus becomes negative or null, i.e. at time $T(u)=$ $\inf \{t>0: U(t) \leq 0\}(T(u)=\infty$ if ruin does not occur). Note that $T(u)$ corresponds to the first-crossing time of the process $\{S(t)\}$ through the upper linear boundary $y=u+c t$. 
Let $\phi(u, t)$ be the probability of non-ruin until time $t$ :

$$
\phi(u, t)=P[T(u)>t]=P[U(\tau)=u+c \tau-S(\tau)>0 \text { for } 0<\tau \leq t],
$$

and let $\psi(u, t)=1-\phi(u, t)$ be the probability of ruin before time $t$. As $t \rightarrow \infty,(2.2)$ becomes the ultimate non-ruin probability $\phi(u)=P[T(u)=\infty]$, the ultimate ruin probability being $\psi(u)=1-\phi(u)$.

In the sequel, we assume that the claim amounts $X_{i}$ are discrete random variables with strictly positive integer values; let $g_{n}=P\left(X_{1}=n\right), n=1,2, \ldots$ Continuous claims might be considered as well but, in practice, claim amounts are usually discretized. For clarity, the initial surplus $u \geq 0$ is assumed to be an integer; extension to real values is straightforward. Finally, operating a time scale change $t \hookrightarrow c t$, we may set in (2.1) $c=1$ and $\lambda=\lambda / c$.

Over any time period $(\tau, \tau+t)$, the aggregate claim size $S(t)$ has a discrete compound Poisson distribution, with Poisson parameter $\lambda t$ and jump sizes $X_{i}$; let $f_{n}(t)=P[S(t)=$ $n], n=0,1, \ldots$ Obviously, the following convolution relations hold: for all $t_{1}, t_{2} \geq 0$,

$$
f_{n}\left(t_{1}+t_{2}\right)=\sum_{j=0}^{n} f_{n-j}\left(t_{1}\right) f_{j}\left(t_{2}\right), \quad n=0,1, \ldots
$$

The easiest way to compute these $f_{n}(t)$, however, is to have recourse to the well-known Panjer recursion formula (see, e.g., Panjer and Willmot [25]); this yields

$$
f_{0}(t)=e^{-\lambda t}, \text { and } f_{n}(t)=\lambda t \sum_{j=1}^{n} \frac{j}{n} g_{j} f_{n-j}(t), \quad n=1,2, \ldots
$$

Different methods are proposed in the literature for determining the probability $\phi(u, t)$.

\section{(1) By discretizing the time scale}

Under this procedure, the original continuous-time scale $t \geq 0$ is replaced by a discretetime scale $t=0,1,2, \ldots$ Then, $\phi(u, t)$ is approximated by

$$
\phi_{a}(u, t)=P[U(\tau)=u+\tau-S(\tau)>0 \text { for } \tau=1,2, \ldots, t] .
$$

Intuitively, such an approximation should be good if the number of checking times for ruin is taken large enough.

(i) De Vylder and Goovaerts [8] apply this procedure to obtain approximated ruin probabilities $\psi_{a}(u, t)=1-\phi_{a}(u, t)$. For uniformity, we consider here the $\phi_{a}(u, t)$. These probabilities are calculated by examining the aggregate claim in the first time period $(0,1)$. This yields the following recursion with respect to $t$ :

$$
\phi_{a}(u, 0)=1, \quad \text { and } \phi_{a}(u, t)=\sum_{j=0}^{u} f_{j}(1) \phi_{a}(u+1-j, t-1), \quad t=1,2, \ldots
$$


A neat truncation procedure is also possible to reduce the number of computations.

(ii) Dickson and Waters [12] propose a different method based again on the relation (2.6). Write $(2.6)$ for $\phi_{a}(u-1, t)$ and isolate now the term $j=0$ in the corresponding right-hand side. This leads to the following recursion with respect to $u$ :

$$
\phi_{a}(u, t)=\left[\phi_{a}(u-1, t+1)-\sum_{j=1}^{u-1} f_{j}(1) \phi_{a}(u-j, t)\right] / f_{0}(1), \quad u=1,2, \ldots
$$

It then remains to evaluate $\phi_{a}(0, t)$, and this is done through formula $(2.15)$ below.

\section{(2) By recursive methods}

Let us return to the original continuous-time scale $t \geq 0$. Claims being discrete, the process $\{S(t)\}$ can reach or cross the straight line $y=u+t$ only at levels $u+1, u+2, \ldots$, so that the only possible meeting-times are $1,2, \ldots$ Thus, the probability $\phi(u, t)$ that there is no such meeting until time $t$, can be expressed under the simplified form

$$
\phi(u, t)=P[S(\tau)<u+\tau \text { for } \tau=1, \ldots,\lfloor t\rfloor, \text { and } S(t)<u+\lfloor t\rfloor+1]
$$

$\lfloor t\rfloor$ representing the integer part of $t$.

Clearly, the probability (2.8) can be expanded as

$$
\phi(u, t)=\sum_{n=0}^{u+\lfloor t\rfloor} P_{n}(t)
$$

where $P_{n}(t) \equiv P_{n}(u, t)$ denotes $P[T(u)>t$ and $S(t)=n]$, i.e.

$$
P_{n}(t)=P[S(\tau)<u+\tau \text { for } \tau=1, \ldots,\lfloor t\rfloor, \text { and } S(t)=n]
$$

Note that $P_{n}(t)=0$ for $n \geq u+\lfloor t\rfloor+1$ (since ruin before time $t$ is then certain). The other $P_{n}(t)$ can now be determined by recursion.

(i) Following Picard and Lefèvre [27], we observe that before time $t$, ruin is impossible when $S(t)=n \leq u$, while for the next values of $n \leq u+\lfloor t\rfloor$, ruin will not occur if it does not happen before time $n-u$. In other words, this yields recursion with respect to $n$ :

$$
P_{n}(t)=\left\{\begin{array}{c}
f_{n}(t), \text { for } 0 \leq n \leq u, \\
\sum_{k=0}^{n-1} P_{k}(n-u) f_{n-k}(t-n+u), \text { for } u+1 \leq n \leq u+\lfloor t\rfloor .
\end{array}\right.
$$

(ii) Rullière and Loisel [30] use a different argument and consider the times $j=$ $0, \ldots,\lfloor t\rfloor$. Similarly to $(2.10)$, put

$$
P_{n}(j)=P[S(\tau)<u+\tau \text { for } \tau=1, \ldots, j, \text { and } S(j)=n]
$$


for $n=0, \ldots, u+j-1$. Examining the aggregate claim during the last time period $(j-1, j)$, we see that $P_{n}(j)$ are given by the following recursion with respect to $j$ :

$$
P_{n}(0)=\delta_{n, 0}, \text { and } P_{n}(j)=\sum_{k=0}^{n} P_{k}(j-1) f_{n-k}(1), \quad j=1, \ldots,\lfloor t\rfloor,
$$

for $n=0, \ldots, u+j-1$. Then, considering the time period $(\lfloor t\rfloor, t), P_{n}(t)$ in $(2.8)$ is obtained by the evident formula

$$
P_{n}(t)=\sum_{k=0}^{n} P_{k}(\lfloor t\rfloor) f_{n-k}(t-\lfloor t\rfloor), \quad n=0, \ldots, u+\lfloor t\rfloor .
$$

\section{(3) Through explicit formulas}

The reader is referred to Lefèvre and Loisel [21] for a unified presentation of closedform formulas. The cases $u=0$ (no initial reserves) and $u>0$ are discussed separately.

(i) When $u=0$, ruin occurs if the process $\{S(t)\}$ reaches or crosses the diagonal line $y=t$. This first-crossing problem has applications in various contexts and is referred to as a ballot problem. By a classical result (see, e.g., Takács [33]), the probability that $S(\tau)$ reaches the level $n$ at time $t \geq n$ without crossing the diagonal line $y=\tau$ is provided by the nice formula

$$
P[S(\tau)<\tau \text { for } 0<\tau \leq t, \text { and } S(t)=n]=\frac{t-n}{t} f_{n}(t), \quad n=0, \ldots,\lfloor t\rfloor .
$$

Therefore, the non-ruin probability $\phi(0, t)$ has the simple expression

$$
\phi(0, t)=\frac{1}{t} \sum_{n=0}^{\lfloor t\rfloor}(t-n) f_{n}(t)
$$

(ii) When $u>0$, one proceeds as in Seal [31] and considers the event $[S(t)<u+t]$. This event can occur in two ways: either there is no ruin before time $t$, or there exists a last time $j$ such that $U(j)=u+j-S(j)=0$, with $j=1, \ldots, u+\lfloor t\rfloor$. Hence,

$$
P[S(t)<u+t]=\phi(u, t)+\sum_{j=1}^{\lfloor t\rfloor} P[S(j)=u+j] P[T(0)>t-j],
$$

so that $\phi(u, t)$ is provided by

$$
\phi(u, t)=\sum_{j=0}^{u+\lfloor t\rfloor} f_{j}(t)-\sum_{j=1}^{u+\lfloor t\rfloor} f_{u+j}(j) \phi(0, t-j),
$$

where $\phi(0, t-j)$ can be calculated from (2.15) above. 
(iii) For $u \geq 0$, Picard and Lefèvre [27] propose a different approach of algebraic essence (see also De Vylder [7]). Firstly, Panjer's recursion (2.4) is considered for any real value of $t$, positive or not. This provides quantities $\tilde{f}_{n}(t), n=0,1, \ldots$, that are defined for all $t \in(-\infty, \infty)$. When $t \geq 0, \tilde{f}_{n}(t)$ reduces to $f_{n}(t)=\tilde{P}[S(t)=n]$, but when $t<0$, $\tilde{f}_{n}(t)$ has no probabilistic interpretation (it can be negative, for example). Nevertheless, an important point is that the convolution relations (2.3) still hold for all reals $t_{1}, t_{2}$. Thanks to this property, one proves that an alternative explicit expression for $\phi(u, t)$ is

$$
\phi(u, t)=\sum_{j=0}^{u}\left\{f_{j}(t)+\tilde{f}_{j}(j-u) \sum_{n=u+1}^{u+\lfloor t\rfloor} \frac{u+t-n}{u+t-j} f_{n-j}(u+t-j)\right\} .
$$

When $u=0,(2.17)$ reduces to (2.15) above

(iv) An additional interest of these explicit formulas is to lead to simple expressions for the ultimate (non-)ruin probabilities. Specifically, let $\mu=E\left(X_{1}\right)$ be the expected claim amount, and assume that $\mu<1$ to guarantee that non-ruin has a positive probability. Passing to the limit $t \rightarrow \infty$ in (2.15), (2.16), (2.17) yields the following formulas:

$$
\begin{aligned}
& \phi(0)=P[T(0)=\infty]=1-\mu ; \\
& \psi(u)=P[T(u)<\infty]=\phi(0) \sum_{j=1}^{\infty} f_{u+j}(j) ; \\
& \phi(u)=P[T(u)=\infty]=\phi(0) \sum_{j=0}^{u} \tilde{f}_{j}(j-u) .
\end{aligned}
$$

(2.19) is a formula of Pollaczeck-Khinchine type (see [2]) and (2.20) is similar to a formula derived in [32]. In practice, the finite sum in (2.20) is easier to handle than the series in (2.19).

Over finite horizon, the different methods for computing $\phi(u, t)$ have their own merits and disadvantages, and none seems to be uniformly better. Numerical comparisons can be found in Dickson [10] for the formulas (2.6), (2.7), (2.16), and in Rullière and Loisel [30] for the formulas (2.9), (2.16), (2.17).

\section{Inhomogeneous premium and claim arrivals}

We start by incorporating non-constancy in the premium input only. Non-stationarity in the claim process will be added later. Such extensions are discussed, e.g., in Picard and Lefèvre [27], Ignatov and Kaishev [16], Ignatov et al. [18] and Lefèvre and Picard [22]. We close with a remark on the definition of ruin.

(1) With non-constant premium function 
Suppose that the premium rate is an arbitrary deterministic function of time, instead of being constant. Then, the cumulated premium income, including the initial reserves, can be represented by a function $h(t)$ that is non-negative and non-decreasing, continuous or not, with $h(0)=u$ and $h(t) \rightarrow \infty$ as $t \rightarrow \infty$. The surplus at time $t$ is given by

$$
U(t)=h(t)-S(t)
$$

which reduces to (2.1) for the classical model where $h(t)=u+c t$. Ruin occurs at the time $T(h)$ when the reserves become negative or null. So, the probability of non-ruin until time $t$, denoted by $\phi(h, t)$, is defined as

$$
\phi(h, t)=P[T(h)>t]=P[U(\tau)=h(\tau)-S(\tau)>0 \text { for } 0<\tau \leq t]
$$

In the present situation, discretizing the time scale as in (2.5) is no longer an efficient method to approximate $\phi(h, t)$. Explicit formulas as in (2.15), (2.16), (2.17) are not available here. The recursive methods of [27] and [30], however, can be easily adapted.

Specifically, let $h^{-1(*)}$ denote the right-continuous inverse of $h$, i.e. $h^{-1(*)}(x)=\inf \{t$ : $h(t)>x\}=\sup \{t: h(t) \leq x\}$. Consider now the following instants:

$$
v_{n}=h^{-1(*)}(n), \quad n=0,1, \ldots,
$$

with thus $v_{0}=\ldots=v_{u-1}=0$. For the classical model, one has $v_{n}=(n-u) / c$ for $n=u, u+1, \ldots$ The instants (3.3) are the only ones to be considered: indeed, claims being discrete, the process $\{S(t)\}$ can reach or cross the upper boundary $h(t)$ only at the levels $v_{u}, v_{u+1}, \ldots$ Thus, denoting by $\lfloor h(t)\rfloor$ the integer part of $h(t),(3.2)$ can be reexpressed under the simplified form

$$
\begin{array}{r}
\phi(h, t)=P\left\{S\left(v_{u}\right)<u, S\left(v_{u+1}\right)<u+1, \ldots, S\left(v_{\lfloor h(t)\rfloor}\right)<\lfloor h(t)\rfloor,\right. \\
\text { and } S(t)<\lfloor h(t)\rfloor+1\} .
\end{array}
$$

As in (2.9), one now writes

$$
\phi(h, t)=\sum_{n=0}^{\lfloor h(t)\rfloor} P_{n}(t),
$$

where $P_{n}(t) \equiv P_{n}^{h}(t)$ denotes $P[T(h)>t$ and $S(t)=n]$, i.e.

$$
P_{n}(t)=P\left\{S\left(v_{u}\right)<u, S\left(v_{u+1}\right)<u+1, \ldots, S\left(v_{\lfloor h(t)\rfloor}\right)<\lfloor h(t)\rfloor, \text { and } S(t)=n\right\} .
$$

(i) Following Picard and Lefèvre [27], we see here that when $S(t)=n$, ruin before time $t$ is only possible if $\lfloor h(t)\rfloor \geq n$, i.e. if $v_{n}<t$, and it will not occur provided it does 
not happen before time $v_{n}$. This leads to the following recursion with respect to $n$ :

$$
P_{n}(t)=\left\{\begin{array}{c}
f_{n}(t), \text { for } 0 \leq n \leq u-1 \\
\sum_{k=0}^{n-1} P_{k}\left(v_{n}\right) f_{n-k}\left(t-v_{n}\right), \text { for } n \geq u \mid v_{n}<t
\end{array}\right.
$$

(ii) Arguing as in Rullière and Loisel [30], define, for $j=0, \ldots,\lfloor h(t)\rfloor-u$,

$$
P_{n}(j)=P\left\{S\left(v_{u}\right)<u, S\left(v_{u+1}\right)<u+1, \ldots, S\left(v_{u+j}\right)<u+j, \text { and } S\left(v_{u+j}\right)=n\right\} .
$$

We first compute these $P_{n}(j)$ by recursion with respect to $j$ :

$$
P_{n}(0)=f_{n}\left(v_{u}\right), \text { and } P_{n}(j)=\sum_{k=0}^{n} P_{k}(j-1) f_{n-k}\left(v_{u+j}-v_{u+j-1}\right),
$$

for $n=0, \ldots, u+j-1$. Then, $P_{n}(t)$ in (3.5) is computed by

$$
P_{n}(t)=\sum_{k=0}^{n} P_{k}(\lfloor h(t)\rfloor-u) f_{n-k}\left(t-v_{\lfloor h(t)\rfloor}\right), \quad n=0, \ldots,\lfloor h(t)\rfloor .
$$

(iii) Picard and Lefèvre [27] propose a simplified algorithm by pointing out that the expression of the $P_{n}(t)$ has a remarkable polynomial structure, apart an exponential factor (see also, e.g., Ignatov and Kaishev [16] and Lefèvre [20]). From (3.7), they prove that

$$
P_{n}(t)=e^{-\lambda t} \bar{A}_{n}\left(t \mid v_{0}, \ldots, v_{n}\right), \quad n=0,1, \ldots
$$

where $\bar{A}_{n}\left(t \mid v_{0}, \ldots, v_{n}\right) \equiv \bar{A}_{n}(t), n=0,1, \ldots$, is a sequence of generalized Appell polynomials (also called Sheffer polynomials) of degree $n$ in $t$ with $\bar{A}_{0}(t)=1$. A short theory about these polynomials can be found, e.g., in Niederhausen [24] and Picard and Lefèvre [26]. We summarize below the elements of direct relevance to our context.

Let $e_{n}(t), n=0,1, \ldots$, be a sequence of polynomials of degree $n$ in $t$ that are of convolution type, i.e. such that for all reals $t_{1}, t_{2}$,

$$
e_{n}\left(t_{1}+t_{2}\right)=\sum_{j=0}^{n} e_{n-j}\left(t_{1}\right) e_{j}\left(t_{2}\right), \quad n=0,1, \ldots
$$

From $(2.3),(2.4)$, we see that in our case, the $e_{n}(t)$ defined by

$$
e_{n}(t)=e^{-\lambda t} f_{n}(t), \quad n=0,1, \ldots
$$

form such a family of polynomials (for all $t$, positive or not).

Now, for a generalized Appell family, each polynomial $\bar{A}_{n}(t)$ admits an expansion of the form

$$
\bar{A}_{n}(t)=\sum_{k=0}^{n} \bar{A}_{k}(0) e_{n-k}(t), \quad n=0,1, \ldots
$$


Note that formula (3.13) can be viewed as a Taylor type expansion of $\bar{A}_{n}(t)$ with respect to the $e_{k}(t)$. The key point in $(3.13)$ is that the coefficients $\bar{A}_{k}(0)$ do not depend on the degree $n$ considered (this property characterizes the family).

It then remains to specify the coefficients $\bar{A}_{k}(0)$. These can be fixed by imposing the border conditions given in (3.7). By that way, we get $\bar{A}_{n}(t)=e_{n}(t)$ for $0 \leq n \leq u-1$ and $\bar{A}_{n}\left(v_{n}\right)=0$ for $n \geq u$, hence the recursion with respect to $n$ :

$$
\bar{A}_{0}(0)=1, \text { and } \bar{A}_{n}(0)=\left\{\begin{array}{c}
0, \text { for } 1 \leq n \leq u-1 \\
-\sum_{k=0}^{n-1} \bar{A}_{k}(0) e_{n-k}\left(v_{n}\right), \text { for } n \geq u
\end{array}\right.
$$

By (3.13), (3.14), the computation of $\bar{A}_{n}(t) \equiv \bar{A}_{n}\left(t \mid v_{0}, \ldots, v_{n}\right)$ is then especially easy and fast, and $P_{n}(t)$ is immediate from (3.10).

\section{(2) With non-stationary claim process}

Suppose that in addition to an arbitrary premium input, claims are ruled by a nonstationary compound Poisson process. So, claims arise according to a Poisson process with non-homogeneous rate $\lambda(t)$ at time $t \geq 0$, and the corresponding claim amounts $\left\{X_{i}(t)\right\}$ are independent and positive integer-valued random variables that have now nonstationary distributions, instead of being equidistributed. Let $g_{n}(t), n=1,2, \ldots$, denote the claim amount distribution for a claim that occurs at time $t \geq 0$.

Over any time period $(\tau, \tau+t)$, the aggregate claim size $S(\tau, \tau+t)$ has a nonhomogeneous discrete compound Poisson distribution; let $f_{n}(\tau, \tau+t)=P[S(\tau, \tau+t)=$ $n], n=0,1, \ldots$ Standard convolution relations give, for all $t_{1}, t_{2} \geq 0$,

$$
f_{n}\left(\tau, \tau+t_{1}+t_{2}\right)=\sum_{j=0}^{n} f_{n-j}\left(\tau, \tau+t_{1}\right) f_{j}\left(\tau+t_{1}, \tau+t_{1}+t_{2}\right), \quad n=0,1, \ldots
$$

Moreover, one finds that

$$
f_{n}(\tau, \tau+t)=e^{-\Lambda(\tau, \tau+t)} \sum_{i_{1}, \ldots, i_{n} \geq 0}^{i_{1}+2 i_{2}+\ldots+n i_{n}=n} \frac{\left[\Lambda_{1}(\tau, \tau+t)\right]^{i_{1}} \ldots\left[\Lambda_{n}(\tau, \tau+t)\right]^{i_{n}}}{i_{1} ! \ldots 1_{n} !}, \quad n=0,1, \ldots
$$

where

$$
\Lambda(\tau, \tau+t)=\int_{\tau}^{\tau+t} \lambda(s) d s, \text { and } \Lambda_{n}(\tau, \tau+t)=\int_{\tau}^{\tau+t} g_{n}(s) \lambda(s) d s, \quad n=1,2, \ldots
$$

Formula (3.16) will enable us to compute these $f_{n}(\tau, \tau+t)$ (a Panjer-type recursion does not seem to be possible here).

Now, the probability $\phi(h, t)$ can still be evaluated using formula (3.4), and it thus remains to calculate the $P_{n}(t)$. This can be done by any of the methods (i), (ii) and (iii) 
above. For instance, the recursion (3.7) becomes here

$$
P_{n}(t)=\left\{\begin{array}{c}
f_{n}(0, t), \text { for } 0 \leq n \leq u-1 \\
\sum_{k=0}^{n-1} P_{k}\left(v_{n}\right) f_{n-k}\left(v_{n}, t\right), \text { for } n \geq u \mid v_{n}<t .
\end{array}\right.
$$

\section{(3) On the definition of ruin}

An alternative definition for ruin is when the reserves become strictly negative, instead of becoming non-positive. So, the non-ruin probability (3.2) is replaced by

$$
\phi(h, t)=P[T(h)>t]=P[U(\tau)=h(\tau)-S(\tau) \geq 0 \text { for } 0<\tau \leq t] .
$$

If $h$ is a continuous function, both definitions (3.2) and (3.18) are equivalent.

Let $h^{-1}$ be the left-continuous inverse of $h$, i.e. $h^{-1}(x)=\inf \{t: h(t) \geq x\}$, and consider the instants

$$
w_{n}=h^{-1}(n), \quad n=0,1, \ldots,
$$

where thus $w_{0}=\ldots=w_{u}=0$. Then, (3.18) can be rewritten as

$$
\begin{aligned}
\phi(h, t)=P\left\{S\left(w_{u}\right) \leq u, S\left(w_{u+1}\right) \leq u+1, \ldots, S\left(w_{\lfloor h(t)\rfloor}\right)\right. & \leq\lfloor h(t)\rfloor, \\
& \text { and } S(t) \leq\lfloor h(t)\rfloor+1\} .
\end{aligned}
$$

Clearly, $\phi(h, t)$ can be computed again from formula (3.4), and any of the methods above will allow us to calculate the $P_{n}(t)$, after suitable adjustment. For instance, the recursion (3.7) is replaced here by

$$
P_{n}(t)=\left\{\begin{array}{c}
f_{n}(t), \text { for } 0 \leq n \leq u \\
\sum_{k=0}^{n} P_{k}\left(w_{n}\right) f_{n-k}\left(t-w_{n}\right), \text { for } n \geq u+1 \mid w_{n} \leq t .
\end{array}\right.
$$

\section{Interdependent claim amounts}

The extended model with interdependent claims is investigated (and applied) in Ignatov and Kaishev [16] and Ignatov et al. [18] (see also Ignatov and Kaishev [17] for continuous claims). Using a slightly different approach, we are going to derive an alternative expression, of similar form, for the non-ruin probability $\phi(h, t)$.

As in Section 3, the premium input is represented by an arbitrary function of time $h(t)$. The successive claim amounts $\left\{X_{i}\right\}$ are still strictly positive integer-valued random variables that are independent of the Poisson process $\{N(t)\}$, but they are no longer assumed to be i.i.d. Specifically, each vector $\left(X_{1}, \ldots, X_{j}\right), j \geq 1$, has now a joint probability distribution with interdependent components. 
Let $S_{j}=X_{1}+\ldots+X_{j}, j \geq 1$, be the partial sums of claim amounts, and denote their joint probability by $h_{s_{1}, \ldots, s_{j}}=P\left(S_{1}=s_{1}, \ldots, S_{j}=s_{j}\right)$, for $1 \leq s_{1}<s_{2}<\ldots<s_{j}$. We suppose hereafter that these distributions are known or, at least, can be estimated.

First step. It is clear that $\phi(h, t)$ can be expanded through (3.5). Claim amounts being interdependent, however, the process $\{S(t)\}$ is no longer a compound Poisson process. This will make the evaluation of $\phi(h, t)$ more lengthy, although not really complicated.

To start, let us condition in (3.5) with respect to the number $N(t)$ of claims during the period $(0, t]$; this gives

$$
\phi(h, t)=e^{-\lambda t} \sum_{n=0}^{\lfloor h(t)\rfloor} \sum_{j=0}^{n} \frac{(\lambda t)^{j}}{j !} P_{n}(t \mid j),
$$

where $P_{n}(t \mid j)$ is the conditional probability

$$
P_{n}(t \mid j)=P[T(h)>t \text { and } S(t)=n \mid N(t)=j], \quad j=0, \ldots, n .
$$

By (4.2), we see that $P_{n}(t \mid 0)=\delta_{n, 0}$, and when $j \geq 1$,

$$
P_{n}(t \mid j)=P\left(S_{j}=n\right), \quad 0 \leq n \leq u-1 .
$$

For the next values $n \geq u$ with $v_{n}<t$, we condition in (4.2) with respect to the amounts of the $j$ claims occurring during $(0, t]$, and with the additional constraint $S(t)=S_{j}=n$; this yields

$$
P_{n}(t \mid j)=\sum_{1 \leq s_{1}<s_{2}<\ldots<s_{j}=n} h_{s_{1}, \ldots, s_{j}} Q_{j}\left(t \mid s_{1}, \ldots, s_{j}\right), \quad j=1, \ldots, n,
$$

where $Q_{j}\left(t \mid s_{1}, \ldots, s_{j}\right)$ is the conditional probability (independent of $n$ )

$$
Q_{j}\left(t \mid s_{1}, \ldots, s_{j}\right)=P\left[T(h)>t \mid N(t)=j ; S_{1}=s_{1}, \ldots, S_{j}=s_{j}\right] .
$$

To evaluate (4.5), let us introduce the following instants, in a similar way to (3.3):

$$
v_{s_{j}}=h^{-1(*)}\left(s_{j}\right), \quad j=1,2, \ldots
$$

Denote by $T_{1}, T_{2}, \ldots$ the successive jump times of the Poisson process $\{N(t)\}$. We then observe that $Q_{j}\left(t \mid s_{1}, \ldots, s_{j}\right)$ can be equivalently represented as

$$
Q_{j}\left(t \mid s_{1}, \ldots, s_{j}\right)=P\left[T_{1}>v_{s_{1}}, \ldots, T_{j}>v_{s_{j}} \mid N(t)=j\right], \quad j=1,2, \ldots
$$

It is well-known that given that a Poisson process has $j$ jumps during $(0, t)$, the vector of jump times $\left(T_{1}, \ldots, T_{j}\right)$ is distributed as the vector of order statistics for an i.i.d. sample 
of $j$ uniform $(0, t)$ random variables. Thus, writing $\left(U_{1: j}, \ldots, U_{j: j}\right)$ for the order statistics of $j$ independent uniform $(0,1)$ r.v., we obtain the following result:

$$
Q_{j}\left(t \mid s_{1}, \ldots, s_{j}\right)=P\left(U_{1: j}>\frac{v_{s_{1}}}{t}, \ldots, U_{j: j}>\frac{v_{s_{j}}}{t}\right) .
$$

By combining (4.1), (4.3) and (4.4), we then get

$$
\begin{aligned}
\phi(h, t)=e^{-\lambda t} & \left\{\delta_{u, 0}+\sum_{n=0}^{u-1} \sum_{j=0}^{n} P\left(S_{j}=n\right) \frac{(\lambda t)^{j}}{j !}\right. \\
& \left.+\sum_{n=u}^{\lfloor h(t)\rfloor} \sum_{j=1}^{n} \lambda^{j} E\left[I\left(S_{j}=n\right) \mathcal{A}_{j}\left(t \mid v_{S_{1}}, \ldots, v_{S_{j}}\right)\right]\right\}
\end{aligned}
$$

where $I$ is an indicator and the expectation is taken with respect to $S_{1}, \ldots, S_{j}$, i.e.

$$
E\left[I\left(S_{j}=n\right) \mathcal{A}_{j}\left(t \mid v_{S_{1}}, \ldots, v_{S_{j}}\right)\right]=\sum_{1 \leq s_{1}<s_{2}<\ldots<s_{j}=n} h_{s_{1}, \ldots, s_{j}} \mathcal{A}_{j}\left(t \mid v_{s_{1}}, \ldots, v_{s_{j}}\right)
$$

and using the notation

$$
\mathcal{A}_{j}\left(t \mid v_{s_{1}}, \ldots, v_{s_{j}}\right)=\frac{t^{j}}{j !} Q_{j}\left(t \mid s_{1}, \ldots, s_{j}\right), \quad j=1,2, \ldots
$$

Second step. (i) The quantities $\mathcal{A}_{j}(t) \equiv \mathcal{A}_{j}\left(t \mid v_{s_{1}}, \ldots, v_{s_{j}}\right)$ remain to be evaluated on the basis of (4.8). One can find in the literature several algorithms for calculating the joint right (or left) tail distributions of the order statistics $\left(U_{1: j}, \ldots, U_{j: j}\right)$ (see, e.g., Denuit et al. [6] and the references therein). In particular, a standard result states that for $0 \leq u_{1} \leq \ldots \leq u_{j} \leq t \leq 1$, the probabilities $a_{j}=P\left(U_{1: j}>u_{1}, \ldots, U_{j: j}>u_{j}\right)$, $j=1,2, \ldots$, satisfy the recurrence relations

$$
a_{0}=1, \quad \text { and } a_{j}=-\sum_{k=0}^{j-1}\left(\begin{array}{l}
j \\
k
\end{array}\right)\left(u_{k}-1\right)^{j-k} a_{k}, \quad j=1,2, \ldots
$$

By (4.8), (4.10), we then deduce for the $\mathcal{A}_{j}(t)$ the following recursion with respect to $j$ :

$$
\mathcal{A}_{0}(t)=1, \quad \text { and } \mathcal{A}_{j}(t)=-\sum_{k=0}^{j-1} \frac{\left(v_{s_{k}}-t\right)^{j-k}}{(j-k) !} \mathcal{A}_{k}(t), \quad j=1,2, \ldots
$$

(ii) In fact, the right tail distributions of $\left(U_{1: j}, \ldots, U_{j: j}\right)$ rely on a simple underlying polynomial structure. More precisely, it can be shown that for $0 \leq u_{1} \leq \ldots \leq u_{j} \leq t \leq 1$,

$$
P\left(U_{1: j}>u_{1}, \ldots, U_{j: j}>u_{j}, \quad \text { and } U_{j: j}<t\right)=j ! A_{j}\left(t \mid u_{1}, \ldots, u_{j}\right), \quad j=1,2, \ldots,
$$

where $A_{j}\left(t \mid u_{1}, \ldots, u_{j}\right) \equiv A_{j}(t), j=0,1, \ldots$, is a sequence of classical Appell polynomials of degree $j$ in $t$ with $A_{0}(t)=1$ (see, e.g., Picard and Lefèvre [26], Denuit et al. [6] and 
Lefèvre [20]). By comparison to the previous generalized Appell polynomials $\bar{A}_{j}(t)$, they correspond to the special case where the convolution type polynomials introduced in (3.11) are the monomials $e_{j}(t)=t^{j} / j$ !. Returning to the $Q_{j}(t)$, (4.8) and (4.13) imply that $Q_{j}\left(t \mid s_{1}, \ldots, s_{j}\right)=A_{j}\left(1 \mid v_{s_{1}} / t, \ldots, v_{s_{j}} / t\right)$. From (4.10), we then deduce the remarkable identity

$$
\mathcal{A}_{j}\left(t \mid v_{s_{1}}, \ldots, v_{s_{j}}\right)=A_{j}\left(t \mid v_{s_{1}}, \ldots, v_{s_{j}}\right), \quad j=0,1, \ldots
$$

So, the $\mathcal{A}_{j}(t)$ can be now determined by adapting the formulas (3.13) and (3.14) to the simple Appell case. This gives

$$
\mathcal{A}_{j}(t)=\sum_{k=0}^{n} \mathcal{A}_{k}(0) \frac{t^{j-k}}{(j-k) !}, \quad j=0,1, \ldots,
$$

where the coefficients $\mathcal{A}_{k}(0)$ do not depend on the degree $j$ (this is the Appell property) and are provided by the recursion with respect to $j$ :

$$
\mathcal{A}_{0}(0)=1, \quad \text { and } \mathcal{A}_{j}(0)=-\sum_{k=0}^{j-1} \mathcal{A}_{k}(0) \frac{v_{j}^{j-k}}{(j-k) !}, \quad j=1,2, \ldots
$$

Remarks. Ignatov and Kaishev [16] present an analogous, but slightly different, expression for $\phi(h, t)$. The difference comes from the starting conditional argument used: they condition the event $[T(h)>t]$ with respect to the claim amounts, while we do it with the event $[T(h)>t$ and $S(t)=n]$. As here, these authors also point out an Appell polynomial structure in their formula (the above coefficients $\mathcal{A}_{k}(0)$ are there given under a determinantal form).

Note that the approach followed here may be applied too if the claim amounts $\left\{X_{i}\right\}$ form an i.i.d. sequence as considered before. For this special case, however, the computational methods reduce to the simpler ones that are described in Section 3, (1).

If $u=0$ and the premium rate is 1, the formula (2.14) given in Section 2, (3) is known to hold also when the claim amounts $\left\{X_{i}\right\}$ are exchangeable r.v.'s (Takács [33]). This can be checked from the previous analysis and using the nice identity (4.17) indicated below. Specifically, let us return to the right tail distribution (4.13) and, instead of the non-decreasing sequence of reals $\left\{u_{1}, \ldots, u_{j}\right\}$, consider now a sequence of r.v. $\left\{U_{1}, \ldots, U_{j}\right\}$ with non-negative exchangeable increments. One can then prove that conditionally on the event $\left(U_{j} \leq t\right)$ where $0 \leq t \leq 1$,

$$
P\left(U_{1: j}>U_{1}, \ldots, U_{j: j}>U_{j}, \quad \text { and } U_{j: j}<t \mid U_{j} \leq t\right)=t^{j-1}\left(t-U_{j}\right), \quad j=1,2, \ldots
$$




\section{Impact of dependence between claim amounts}

It is often believed that positive dependence between risks increases the probability of ruin over any given time horizon. This seems to be natural, for example, if the different claims are subjected to some exterior environment. Conclusions in that direction are indeed pointed out in, e.g., Cossette and Marceau [5], Frostig [13] and Picard et al. [28].

In this Section, we will firstly show that ruin probabilities can, not only increase, but also diminish owing to the presence of positive dependence between claim amounts. Such a decreasing effect is possible in a different model where each claim size depends on the previous claim interval (as, e.g., in Albrecher and Boxma [?] and Boudreault et al. [3]), since positive dependence here corresponds to a kind of mutualisation that will play a protective role. On the other hand, the decreasing effect obtained through our model comes rather from the claim size distribution itself as it is a consequence of the max-sumequivalence property for heavy-tailed distributions. Secondly, we will establish a result stating that for certain heavy-tailed claim amount laws, positive dependence affects ruin probabilities in a monotone way, increasing or decreasing, when the initial surplus is large enough. For related questions on the asymptotic tail behaviour of sums of dependent risks, the reader is referred, e.g., to Wüthrich [34] and Albrecher et al. [1].

\section{(1) Possible effects of positive dependence}

To illustrate possible effects of positive dependence, we consider two particular risk models in which the successive claim amounts $X_{i}, i \geq 1$, have the same distributions but are interdependent in a comonotonic way. More precisely, for both models, we will compare the ruin probability $\psi(u, t)$ in the independent case, i.e. when the $X_{i}$ are i.i.d., and in a comonotonic case when all the $X_{i}=X_{1}$ almost surely, i.e. under an extremal positive dependence.

(i) Let us assume that the successive claim amounts have a common biatomic distribution given by

$$
P\left(X_{1}=1\right)=0.99 \text { and } P\left(X_{1}=1000\right)=0.01
$$

Note that this law can be considered as heavy-tailed. Take $\lambda=c=1$, choose an horizon of length $t=10$ and let $u=990$ be the initial surplus.

Intuitively, as the average number of claims up to $t$ is equal to $\lambda t=10$, ruin will occur when $u=990$ if there arises (at least) one large claim (of size 1000) before time $t$, or if there arise sufficiently many small claims (of size 1), this event being however of small probability. In addition, the probability of getting at least one large claim is clearly smaller higher in the comonotonic case than in the independent case. Thus, one expects that the ruin probability before time $t=10$ will be also smaller in the comonotonic case. 
Let us show this rigourously. By definition,

$$
\psi(990,10)=P[S(\tau) \leq 990+\tau \text { for some } \tau \leq 10]
$$

From $(5.1)$ and since $P[N(10) \geq 991]<10^{-500}$ is negligible, we can approximate $\psi(990,10)$ by a quantity $\psi_{a}(990,10)$ defined by

$$
\psi_{a}(990,10)=\sum_{j=1}^{990} P[N(10)=j \text { and at least one these } \mathrm{j} \text { claims is of size 1000]. }
$$

In the comonotonic case, (5.2) yields the approximation $\psi_{a}^{\text {com }}(990,10)$ given by

$$
\psi_{a}^{\text {com }}(990,10)=P\left(X_{1}=1000\right) P[1 \leq N(10) \leq 990],
$$

while in the independent case, the corresponding approximation $\psi_{a}^{\perp}(990,10)$ is

$$
\psi_{a}^{\perp}(990,10)=\sum_{j=1}^{990}\left[1-P\left(X_{1}=1\right)^{j}\right] P[N(10)=j] .
$$

We so see that

$$
\begin{aligned}
{\left[\psi_{a}^{\perp}-\psi_{a}^{c o m}\right](990,10) } & >P[2 \leq N(10) \leq 990]\left\{\left[1-P\left(X_{1}=1\right)^{2}\right]-P\left(X_{1}=1000\right)\right\} \\
& \simeq 0.00227>>10^{-500}>P[N(10) \geq 991] .
\end{aligned}
$$

Thus, as for the exact ruin probabilities $\psi^{\perp}$ and $\psi^{\text {com }}$, we get the inequality $\psi^{\perp}(990,10)>$ $\psi^{\text {com }}(990,10)$.

(ii) Let us consider another situation where the common claim amount distribution is still a biatomic law but given now by

$$
P\left(X_{1}=1\right)=0.99 \quad \text { and } \quad P\left(X_{1}=10\right)=0.01
$$

In comparison with (5.1), this law may be viewed as light-tailed. Take again $\lambda=c=1$ and $t=10$, and set here $u=100$ as initial surplus.

This time, large claims (of size 10) will cause ruin before time $t=10$ only if they are also relatively numerous, which is more probable in the comonotonic case. So, one expects intuitively that the comonotonic case could provide a higher ruin probability than the independent case.

Let us establish this result. First, we observe that ruin is sure when there arise 11 large claims before $t=10$. Thus, the ruin probability for the comonotonic case, $\psi^{\text {com }}(100,10)$, satisfies

$$
\psi^{\text {com }}(100,10)>P[N(10) \geq 11] P\left(X_{1}=10\right) \simeq 0.00417
$$


On the other hand, occurrence of ruin before $t=10$ implies necessarily that the the total claim amount at $t$ is larger than $u=100$. Using (5.3), we then have

$$
\psi(100,10) \leq P[S(10)>100]=1-\sum_{j=0}^{100} P[N(10)=j, S(10) \leq 100] .
$$

For $0 \leq j \leq 10$, the event $[N(10)=j, S(10) \leq 100]$ is equivalent to $[N(10)=j]$. For $11 \leq j \leq 100$, the event $[N(10)=j, S(10) \leq 100]$ means that the number of large claims, $k$ say, satisfies the relation $10 k+(j-k) \leq 100$; so, in the independent case,

$P[N(10)=j, S(10) \leq 100]=P[N(10)=j] \sum_{k=0}^{\lfloor(100-j) / 9\rfloor}\left(\begin{array}{l}j \\ k\end{array}\right)\left[P\left(X_{1}=10\right)\right]^{k}\left[P\left(X_{1}=1\right)\right]^{j-k}$.

Thus, the ruin probability for the i.i.d. model, $\psi^{\perp}(100,10)$, satisfies

$$
\begin{aligned}
\psi^{\perp}(100,10) \leq & 1-\sum_{j=0}^{100} P[N(10)=j] \\
& \left.\left(\sum_{k=0}^{\lfloor(100-j) / 9\rfloor}\left(\begin{array}{l}
j \\
k
\end{array}\right)\left[P\left(X_{1}=10\right)\right]^{k} P\left(X_{1}=1\right)\right]^{j-k}\right) \simeq 10^{-14} .
\end{aligned}
$$

Comparing (5.4) with (5.5) then gives the inequality $\psi^{\perp}(100,10)<\psi^{\text {com }}(100,10)$.

In general, positive dependence does not have affect ruin probabilities in a monotonic way. Nevertheless, the two examples above show that asymptotically as $u \rightarrow \infty$, such a property could be true for certain classes of interdependent claim amounts with heavytailed distributions, as in example (i), or with light-tailed distributions, as in example (ii).

\section{(2) A monotonicity result for heavy-tailed laws}

In this Section, we are going to prove that for certain heavy-tailed models, a stronger positive dependence between claim amounts can either increase or decrease ruin probabilities when the initial surplus is large enough. Various extensions of this result are possible and will be presented in a forthcoming paper.

Let us assume that the successive claim amounts $\left\{X_{i}\right\}$ are represented as follows:

$$
X_{i}=I_{i} W_{0}+\left(1-I_{i}\right) W_{i}, \quad i \geq 1,
$$

where $I_{i}, i \geq 1$, is a sequence of i.i.d. Bernoulli random variables with parameter $p=$ $P\left(I_{1}=1\right) \in[0,1]$ and $W_{i}, i \geq 0$, is a sequence of i.i.d. positive random variables with Pareto distribution given by $P\left(W_{0}>x\right)=x^{-\alpha}, x \geq 1$, where $\alpha>0$, these two sequences being independent. Obviously, the $X_{i}$ are positively dependent through the common factor $W_{0}$ and their interdependence becomes stronger when $p$ increases. 
Denote $S(t) \equiv S^{p}(t)$ and $\psi^{p}(u, t) \equiv \psi^{p}(u, t)$. Our result states that if $u$ is large enough, then for any $c, t>0$,

$$
p_{1}<p_{2} \text { implies } \psi^{p_{1}}(u, t)>\psi^{p_{2}}(u, t) \text { when } \alpha<1
$$

while the inverse inequality holds, i.e. $\psi^{p_{1}}(u, t)<\psi^{p_{2}}(u, t)$, when $\alpha>1$.

Proof of (5.7). A key step of the proof is the max-sum-equivalence property (see, e.g., Cai and Tang [4]). Two random variables $Y_{1}, Y_{2}$ are said to satisfy that property if

$$
P\left(Y_{1}+Y_{2}>x\right) \sim P\left(Y_{1}>x\right)+P\left(Y_{2}>x\right)
$$

In the independent case, this is equivalent to

$$
P\left(Y_{1}+Y_{2}>x\right) \sim P\left[\max \left(Y_{1}, Y_{2}\right)>x\right]
$$

For the model (5.6), the $W_{i}, i \geq 0$, are i.i.d. and with regularly varying tail of exponent $\alpha$. So, for any $j \geq 1$ and any pairwise distinct $i_{1}, \ldots, i_{j} \geq 1$,

$$
P\left(W_{i_{1}}+\ldots+W_{i_{j}}>x\right) \sim P\left[\max \left(X_{i_{1}}, \ldots, X_{i_{j}}\right)>x\right]=1-\left(1-x^{-\alpha}\right)^{j}
$$

whence

$$
P\left(W_{i_{1}}+\ldots+W_{i_{j}}>x\right)=P\left(W_{1}+\ldots+W_{j}>x\right) \sim j x^{-\alpha} .
$$

We also have, for any $k>0$,

$$
P\left(k W_{0}>x\right)=P\left(W_{0}>x / k\right) \sim k^{\alpha} x^{-\alpha}
$$

Using (5.8) and (5.9), we find that for any $j \geq 1$ and $0 \leq k \leq j$,

$$
\begin{aligned}
P\left[S^{p}(t)>\right. & \left.x \mid N(t)=j, I_{j_{1}}=\ldots=I_{j_{j-k}}=0, I_{j_{j-k+1}}=\ldots=I_{j_{j}}=1\right] \\
& =P\left[\left(W_{1}+\cdots+W_{k-j}\right)+\left(k W_{0}\right)>x\right] \sim\left(k-j+j^{\alpha}\right) x^{-\alpha}
\end{aligned}
$$

for $j=0, P\left[S^{p}(t)>x \mid N(t)=0\right]=0$. By the independence assumptions made above, we then deduce from (5.10) that

$$
P\left[S^{p}(t)>x\right] \sim\left\{\sum_{j=1}^{\infty} e^{-\lambda t} \frac{(\lambda t)^{j}}{j !}\left[\sum_{k=0}^{j}\left(\begin{array}{l}
j \\
k
\end{array}\right) p^{k}(1-p)^{j-k}\left(j-k+k^{\alpha}\right)\right]\right\} x^{-\alpha} .
$$

This formula can be rewritten as

$$
P\left[S^{p}(t)>x\right] \sim\left\{\lambda t+E\left[\left(Z^{p}(t)\right)^{\alpha}-Z^{p}(t)\right]\right\} x^{-\alpha},
$$

where $Z^{p}(t)$ denotes a binomial random variable $\operatorname{Bin}[N(t), p]$. 
Note that for $u$ large enough,

$$
\psi^{p}(u, t) \sim P\left[S^{p}(t)>u+c t\right], \text { for any } c, t>0,
$$

$(\sim$ meaning that their ratio tends to 1 as $u \rightarrow \infty)$. Indeed,

$$
\begin{aligned}
0 & \leq \frac{\psi^{p}(u, t)-P\left[S^{p}(t)>u+c t\right]}{\psi^{p}(u, t)} \leq \frac{\psi^{p}(u, t)-P\left[S^{p}(t)>u+c t\right]}{P\left[S^{p}(t)>u+c t\right]} \\
& \leq \frac{P\left[S^{p}(t)>u\right]-P\left[S^{p}(t)>u+c t\right]}{P\left[S^{p}(t)>u+c t\right]} \rightarrow 0 \text { as } u \rightarrow \infty \text { from 5.11. }
\end{aligned}
$$

Therefore, from (5.12) and (5.11), we obtain that for any $c, t>0$,

$$
\psi^{p_{1}}(u, t)-\psi^{p_{2}}(u, t) \sim\left\{E\left[\left(Z^{p_{1}}(t)\right)^{\alpha}-Z^{p_{1}}(t)\right]-E\left[\left(Z^{p_{2}}(t)\right)^{\alpha}-Z^{p_{2}}(t)\right]\right\} x^{-\alpha} .
$$

The binomial law $\operatorname{Bin}[N(t), p]$ is known to be stochastically increasing in the parameter $p$ (see, e.g., Lefèvre and Utev [23]). Since the function $f(x)=x^{\alpha}-x, x \in\{0,1, \ldots\}$, is decreasing (resp. increasing) when $\alpha<1$ (resp. $\alpha>1$ ), we then deduce the announced implication (5.7). $\diamond$

\section{Acknowledgements}

This work was partly conducted while Cl. Lefèvre was visiting the Institut de Science Financière et d'Assurances (I.S.F.A.), Université Claude Bernard Lyon 1. His sincere thanks go to all the members of the Institut for their warm welcome and many interesting discussions.

\section{References}

[1] Albrecher, H., Asmussen, S. and Kortschak, D. (2006). Tail asymptotics for the sum of two heavy-tailed dependent risks, Extremes 9, 107-130.

[2] Asmussen, S. (2000). Ruin Probabilities, World Scientific, Singapore.

[3] Boudreault, M., Cossette, H., Landriault, D. and Marceau, E. (2006). On a risk model with dependence between interclaim arrivals and claim sizes, Scandinavian Actuarial Journal 5, 265-285.

[4] Cai, J. and Tang, Q. (2004). On max-sum equivalence and convolution closure of heavy-tailed distributions and their applications, Journal of applied Probability $\mathbf{4 1}$, 117-130.

[5] Cossette, H. and Marceau, E. (2000). The discrete-time risk model with correlated classes of business, Insurance: Mathematics and Economics 26, 133-149.

[6] Denuit, M. , Lefèvre, C. and Picard, P. (2003). Polynomial structures in order statistics distributions, Journal of Statistical Planning and Inference 113, 151-178. 
[7] De Vylder, F.E. (1999). Numerical finite-time ruin probabilities by the Picard-Lefèvre formula, Scandinavian Actuarial Journal, 97-105.

[8] De Vylder, F.E. and Goovaerts, M.J. (1988). Recursive calculation of finite-time ruin probabilities, Insurance: Mathematics and Economics 7, 1-7.

[9] Dickson, D.C.M. (1992). On the distribution of surplus prior to ruin, Insurance: Mathematics and Economics 11, 191-207.

[10] Dickson, D.C.M., (1999). On numerical evaluation of finite time survival probabilities, British Actuarial Journal 5, 575-584.

[11] Dickson, D.C.M., (2005). Insurance Risk and Ruin, Cambridge University Press, Cambridge.

[12] Dickson, D.C.M. and Waters, H.R. (1991). Recursive calculation of survival probabilities, ASTIN Bulletin 21, 199-221.

[13] Frostig, E. (2003). Ordering ruin probabilities for dependent claim streams, Insurance: Mathematics and Economics 32, 93-114.

[14] Gerber, H.U. (1979). An Introduction to Mathematical Risk Theory, S.S. Huebner Foundation Monograph, University of Philadelphia, Philadelphia.

[15] Grandell, J. (1990). Aspects of Risk Theory, Springer-Verlag, New York.

[16] Ignatov, Z.G. and Kaishev, V.K. (2000). Two-sided bounds for the finite time probability of ruin, Scandinavian Actuarial Journal, 46-62.

[17] Ignatov, Z.G. and Kaishev, V.K. (2004). A finite-time ruin probability formula for continuous claim severities, Journal of Applied Probability 41, 570-578

[18] Ignatov, Z.G., Kaishev, V.K. and Krachunov, R.S. (2001). An improved finite-time ruin probability formula and its Mathematica implementation, Insurance: Mathematics and Economics 29, 375-386.

[19] Kaas, R., Goovaerts, M.J., Dhaene, J. and Denuit, M. (2001). Modern Actuarial Risk Theory, Kluwer, Dordrecht.

[20] Lefèvre, C. (2007). Discrete compound Poisson process with curved boundaries: polynomial structures and recursions, Mehodology and Computing in Applied Probability 9, 243-262.

[21] Lefèvre, C. and Loisel, S. (2007). On finite-time ruin probabilities for classical risk models, Scandinavian Actuarial Journal, to appear.

[22] Lefèvre, C. and Picard, P. (2006). A nonhomogeneous risk model for insurance, Computers and Mathematics with Applications 51,325-334.

[23] Lefèvre, C. and Utev, S. (1996). Comparing sums of exchangeable Bernoulli random variables, Journal of Applied Probability 33, 285-310. 
[24] Niederhausen, H. (1981). Sheffer polynomials for computing exact KolmogorovSmirnov and Rényi type distributions, Annals of Statistics 9, 923-944.

[25] Panjer, H.H. and Willmot, G.E. (1992). Insurance Risk Models, Society of Actuaries, Schaumburg.

[26] Picard, P. and Lefèvre, C. (1996). First crossing of basic counting processes with lower non-linear bundaries: a unified approach through pseudopolynomials (I), Advances in Applied Probability 28, 853-876.

[27] Picard, P. and Lefèvre, C. (1997). The probability of ruin in finite time with discrete claim size distribution, Scandinavian Actuarial Journal, 58-69.

[28] Picard, P., Lefèvre, C. and Coulibaly, I. (2003). Multirisks model and finite-time ruin probabilities, Methodology and Computing in Applied Probability 5, 337-353.

[29] Rolski, T., Schmidli, H., Schmidt, V. and Teugels, J. (1999). Stochastic Processes for Insurance and Finance, Wiley, Chichester.

[30] Rullière, D. and Loisel, S. (2004). Another look at the Picard-Lefèvre formula for finite-time ruin probabilities, Insurance: Mathematics and Economics 35, 187-203.

[31] Seal, H.L. (1969). The Stochastic Theory of a Risk Business, Wiley, New York.

[32] Shiu, E.S.W. (1988). Calculation of the probability of eventual ruin by Beekman's convolution series, Insurance: Mathematics and Economics 7, 41-47.

[33] Takács, L. (1967). Combinatorial Methods in the Theory of Stochastic Processes, Wiley, New York.

[34] Wüthrich, M.V. (2003). Asymptotic value-at-risk estimates for sums of dependent random variables, ASTIN Bulletin 33, 75-92. 